\title{
The Hilbert-Type Integral Inequalities with a Homogeneous Kernel of $-\lambda$-Degree
}

\author{
Wuyi Zhong \\ Department of Mathematics, Guangdong Institute of Education, Guangzhou, Guangdong 510303, China
}

Correspondence should be addressed to Wuyi Zhong, wp@bao.ac.cn

Received 24 March 2008; Accepted 19 May 2008

Recommended by Ondrej Dosly

By introducing an integral operator, a norm with a weight function, and two pairs of conjugate exponents, we find the conditions for the Hilbert-type integral inequalities with a homogeneous kernel of $-\lambda$-degree. We also prove that the constant factors in the inequalities are all the best possible. As the particular situations, some new inequalities with a homogeneous kernel and their other two forms are given. We extend some previous results.

Copyright (C) 2008 Wuyi Zhong. This is an open access article distributed under the Creative Commons Attribution License, which permits unrestricted use, distribution, and reproduction in any medium, provided the original work is properly cited.

\section{Introduction and preliminaries}

If $p>1,(1 / p)+(1 / q)=1, f(x), g(x) \geq 0, f \in L^{p}(0, \infty)$, and $g \in L^{q}(0, \infty)$, such that $0<$ $\left(\int_{0}^{\infty} f^{p}(x) d x\right)^{1 / p}<\infty$ and $0<\left(\int_{0}^{\infty} g^{q}(y) d y\right)^{1 / p}<\infty$, then we have

$$
\iint_{0}^{\infty} \frac{f(x) g(y)}{x+y} d x d y<\frac{\pi}{\sin (\pi / p)}\left(\int_{0}^{\infty} f^{p}(x) d x\right)^{1 / p}\left(\int_{0}^{\infty} g^{q}(y) d y\right)^{1 / p},
$$

where the constant factor $\pi /(\sin (\pi / p))$ is the best possible. Equation (1.1) is the famous Hardy-Hilbert inequality proved by Hardy et al. [1].

Let $K(x, y)=1 /(x+y),(T f)(y):=\int_{0}^{\infty} K(x, y) f(x) d x$, and $\|f\|_{p}:=\left\{\int_{0}^{\infty}|f(x)|^{p} d x\right\}^{1 / p}$ (or $(T g)(x):=\int_{0}^{\infty} K(x, y) g(y) d y$ and $\left.\|g\|_{q}:=\left\{\int_{0}^{\infty}|g(y)|^{q} d y\right\}^{1 / q}\right)$. Yang [2] rewrote (1.1) as

$$
(T f, g)<\frac{\pi}{\sin (\pi / p)}\|f\|_{p}\|g\|_{q}
$$

where $T: L^{r}(0, \infty) \rightarrow L^{r}(0, \infty)(r=p, q)$ is an integral operator, $(T f, g)=\int_{0}^{\infty}\left(\int_{0}^{\infty} K(x\right.$, $y) f(x) d x) g(y) d y=\iint_{0}^{\infty} K(x, y) f(x) g(y) d x d y=(T g, f)$ is the formal inner product of $T f$ and $g,\|f\|_{p}\left(\right.$ or $\left.\|g\|_{q}\right)$ is the norm of function $f$ in $L^{p}(0, \infty)$ (or function $g$ in $L^{q}(0, \infty)$ ). 
If $K(x, y)$ is a real measurable function and satisfies $K(u x, u y)=u^{-\lambda} K(x, y)(\lambda>0, u>$ $0)$ for $(x, y) \in(0, \infty) \times(0, \infty)$, then $K(x, y)$ is called a homogeneous function of $-\lambda$-degree. Hence, $K(x, y)=1 /(x+y)$ in (1.2) is called a homogeneous kernel function of the integral operator $T$ and inequality (1.1) is called an inequality with the homogeneous kernel of -1degree also.

Recently, inequality (1.1) has been extended by introducing some parameters [3-6]. A reverse Hilbert-Pachpatte inequality was first proved by Zhao and Debnath [7]. Zhong and Yang $[8,9]$ gave some reverse inequalities concerning some extensions of (1.1). About the Hilbert-type integral inequalities with a symmetric homogeneous kernel, Krnić and Pečarić have researched [10]. Zhong and Yang found the conditions for the multiple Hilbert-type integral inequalities with a symmetric kernel [11]. Xie got a Hilbert-type integral inequality with an unsymmetrical homogeneous kernel of -3-degree [12]. Xie's work, as we will see in Remark 3.3, is a special situation of our results for $\lambda=3$ and $s=r=2$.

By introducing an integral operator $T$, a norm $\|f\|_{p, \omega}$ with a weight function and two pairs of conjugate exponents $(p, q),(r, s)$ in this paper, we find some conditions under which the Hilbert-type integral inequalities with a homogeneous kernel of $-\lambda$-degree and their reverse forms and equivalent forms hold, here the homogeneous kernels $K(x, y)$ can be unsymmetrical. We also prove that the constant factors in these inequalities are all the best possible. As applications of the theorems, some new inequalities with a homogeneous kernel and their other two forms are given.

For these purposes, we introduce some notations as follows.

If $p>1,(1 / p)+(1 / q)=1$ and $r>1,(1 / r)+(1 / s)=1$, a norm of $f$ with the weight function $\omega(x)$ is defined by

$$
\|f\|_{p, \omega}:=\left\{\int_{0}^{\infty} \omega(x)|f(x)|^{p} d x\right\}^{1 / p}
$$

where $f(x), \omega(x) \geq 0$ are measurable in $(0, \infty)$. If $\|f\|_{p, \omega}<\infty$, then it is marked by $f \in L_{\omega}^{p}\left(\mathbb{R}_{+}\right)$ (for $0<p<1$ or $q<0$, we still use the formal mark (1.3) in the following).

Supposing that $K(x, y) \geq 0$ is a measurable function in $(0, \infty) \times(0, \infty)$ and defining an integral operator $T$, for $f, g \geq 0$,

$$
\begin{aligned}
& (T f)(y):=\int_{0}^{\infty} K(x, y) f(x) d x \quad(y \in(0, \infty)), \\
& (T g)(x):=\int_{0}^{\infty} K(x, y) g(y) d y \quad(x \in(0, \infty)),
\end{aligned}
$$

then we have the formal inner as follows:

$$
(T f, g)=(T g, f)=\iint_{0}^{\infty} K(x, y) f(x) g(y) d x d y .
$$

Lemma 1.1 (cf. [13]). Assume that $p>0,(1 / p)+(1 / q)=1, F, G \geq 0$, and $F \in L^{p}(E), G \in L^{q}(E)$. One has the following Hölder inequalities:

(1) if $p>1$, then

$$
\int_{E} F(t) G(t) d t \leq\left(\int_{E} F^{p}(t) d t\right)^{1 / p}\left(\int_{E} G^{q}(t) d t\right)^{1 / p},
$$


(2) if $0<p<1$, then

$$
\int_{E} F(t) G(t) d t \geq\left(\int_{E} F^{p}(t) d t\right)^{1 / p}\left(\int_{E} G^{q}(t) d t\right)^{1 / p}
$$

where the equalities hold if and only if there exist real numbers $A$ and $B\left(A^{2}+B^{2} \neq 0\right)$, such that $A F^{p}(t)=B G^{q}(t)$ a.e. in $E$.

Lemma 1.2. If $s>1,(1 / r)+(1 / s)=1, p>0, p \neq 1,(1 / p)+(1 / q)=1, \lambda>0$, setting that $K(x, y)(\geq 0)$ is a homogeneous kernel function of-l-degree, and defining that

$$
\begin{gathered}
\omega_{1}(\lambda, s, x):=\int_{0}^{\infty} K(x, y) \frac{x^{\lambda / r}}{y^{1-(\lambda / s)}} d y, \\
\omega_{2}(\lambda, r, y):=\int_{0}^{\infty} K(x, y) \frac{y^{\lambda / s}}{x^{1-(\lambda / r)}} d x, \\
C_{\lambda}(s):=\int_{0}^{\infty} K(1, u) u^{(\lambda / s)-1} d u,
\end{gathered}
$$

then one has

$$
\omega_{1}(\lambda, s, x)=\omega_{2}(\lambda, r, y)=C_{\lambda}(s)
$$

Proof. By the $-\lambda$ homogeneity of the kernel $K(x, y)$, for $x>0$, and setting $u=y / x$, we have

$$
\omega_{1}(\lambda, s, x)=\int_{0}^{\infty} K\left(x, x \frac{y}{x}\right) \frac{x^{(\lambda / r)+1}}{(x(y / x))^{1-(\lambda / s)}} d \frac{y}{x}=\int_{0}^{\infty} K(1, u) u^{(\lambda / s)-1} d u=C_{\lambda}(s)
$$

and for $y>0$, letting $x=y / u$, by $d x=-\left(y / u^{2}\right) d u$, it is easy to find that

$$
\omega_{2}(\lambda, r, y)=\int_{0}^{\infty} K\left(\frac{y}{u}, y\right) \frac{y^{\lambda / s}\left(y / u^{2}\right)}{y^{1-(\lambda / r)} u^{(\lambda / r)-1}} d u=\int_{0}^{\infty} K(1, u) u^{(\lambda / s)-1} d u
$$

equation (1.12) is valid. The lemma is proved.

Lemma 1.3. If $s>1,(1 / r)+(1 / s)=1, p>0, p \neq 1,(1 / p)+(1 / q)=1, \lambda>0$, suppose that

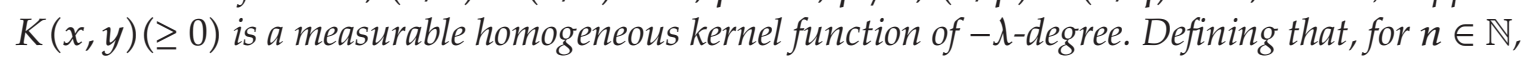

$$
I_{n}=\int_{1}^{\infty} x^{-1-(1 / n)}\left[\int_{1}^{\infty} K(x, y) \frac{x^{(\lambda / r)+(1 / n q)}}{y^{1-(\lambda / s)+(1 / n q)}} d y\right] d x
$$

one has

$$
I_{n}=n\left[\int_{0}^{1} K(1, u) u^{(\lambda / s)-1+(1 / n p)} d u+\int_{1}^{\infty} K(1, u) u^{(\lambda / s)-1-(1 / n q)} d u\right]
$$


Proof. By the $-\lambda$ homogeneity of the kernel $K(x, y)$, for $x>1$ and setting $u=y / x$, we have

$$
\int_{1}^{\infty} K(x, y) \frac{x^{(\lambda / r)+(1 / n q)}}{y^{1-(\lambda / s)+(1 / n q)}} d y=\int_{1 / x}^{1} K(1, u) u^{(\lambda / s)-1-(1 / n q)} d u+\int_{1}^{\infty} K(1, u) u^{(\lambda / s)-1-(1 / n q)} d u
$$

It follows that

$$
I_{n}=n \int_{1}^{\infty} K(1, u) u^{(\lambda / s)-1-(1 / n q)} d u+\int_{1}^{\infty} x^{-1-(1 / n)} d x \int_{1 / x}^{1} K(1, u) u^{(\lambda / s)-1-(1 / n q)} d u
$$

By the nonnegative measurability of $K(x, y)$ and using Tonelli theorem [14], we have

$$
\begin{aligned}
& \int_{1}^{\infty} x^{-1-(1 / n)} d x \int_{(1 / x)}^{1} K(1, u) u^{(\lambda / s)-1-(1 / n q)} d u \\
& \quad=\int_{0}^{1} K(1, u) u^{(\lambda / s)-1-(1 / n q)} d u \int_{(1 / u)}^{\infty} x^{-1-(1 / n)} d x=n \int_{0}^{1} K(1, u) u^{(\lambda / s)-1+(1 / n p)} d u .
\end{aligned}
$$

In view of (1.18) and (1.19), we have (1.16). The lemma is proved.

\section{Main results}

Theorem 2.1. Supposing $p>1,(1 / p)+(1 / q)=1, s>1,(1 / r)+(1 / s)=1, \lambda>0, f, g \geq 0$, letting $\omega(x)=x^{p(1-(\lambda / r))-1}, \varpi(y)=y^{q(1-(\lambda / s))-1}, h(y)=y^{(p \lambda / s)-1}$, and setting $T$ is a integral operator defined by (1.4) (or (1.5)), if the following conditions are satisfied:

(1) $K(x, y) \geq 0$ is a measurable homogeneous kernel function of- $\lambda$-degree,

(2) the weight coefficient $C_{\lambda}(s)=\int_{0}^{\infty} K(1, u) u^{(\lambda / s)-1} d u$ is a positive number depending only on the parameters $\lambda, s$, then one has

If $f \in L_{\omega}^{p}\left(\mathbb{R}_{+}\right), g \in L_{\varpi}^{q}\left(\mathbb{R}_{+}\right)$, and $\|f\|_{p, \omega},\|g\|_{q, \omega}>0$, then

$$
(T f, g)=\iint_{0}^{\infty} K(x, y) f(x) g(y) d x d y<C_{\lambda}(s)\|f\|_{p, \omega}\|g\|_{q, \varpi}
$$

if $f \in L_{\omega}^{p}\left(\mathbb{R}_{+}\right)$and $\|f\|_{p, \omega}>0$, then $T f \in L_{h}^{p}\left(\mathbb{R}_{+}\right)$and

$$
\|T f\|_{p, h}=\left\{\int_{0}^{\infty} y^{(p \lambda / s)-1}\left(\int_{0}^{\infty} K(x, y) f(x) d x\right)^{p} d y\right\}^{1 / p}<C_{\lambda}(s)\|f\|_{p, \omega},
$$

where the same constant factor $C_{\lambda}(s)$ in (2.1) and (2.2) is the best possible. Inequalities (2.1) and (2.2) are equivalent. 
Proof. (1) By $p>1$ and the nonnegative measurability of $K(x, y)$, we can use Hölder's inequality (1.10) and Tonelli theorem in the following:

$$
\begin{aligned}
(T f, g)= & \iint_{0}^{\infty}\left[K^{(1 / p)}(x, y) f(x) \frac{x^{(1 / q)(1-(\lambda / r))}}{y^{(1 / p)(1-(\lambda / s))}}\right]\left[K^{1 / q}(x, y) g(y) \frac{y^{(1 / p)(1-(\lambda / s))}}{x^{(1 / q)(1-(\lambda / r))}}\right] d x d y \\
\leq & \left\{\iint_{0}^{\infty} K(x, y) \frac{x^{(p-1)(1-(\lambda / r))}}{y^{1-(\lambda / s)}} f^{p}(x) d y d x\right\}^{1 / p} \\
& \times\left\{\iint_{0}^{\infty} K(x, y) \frac{y^{(q-1)(1-(\lambda / s))}}{x^{1-(\lambda / r)}} g^{q}(y) d x d y\right\}^{1 / q} \\
= & \left\{\int_{0}^{\infty} \omega_{1}(\lambda, s, x) x^{p(1-(\lambda / r))-1} f^{p}(x) d x\right\}^{1 / p}\left\{\int_{0}^{\infty} \omega_{2}(\lambda, r, y) y^{q(1-(\lambda / s))-1} g^{q}(y) d y\right\}^{1 / q},
\end{aligned}
$$

where $\omega_{1}(\lambda, s, x), \omega_{2}(\lambda, r, y)$ are defined by (1.9) and (1.10), respectively. Using (1.12) and condition (2), we have

$$
(T f, g) \leq C_{\lambda}(s)\|f\|_{p, \omega}\|g\|_{q, \varpi}
$$

If (2.3) takes the form of equality, then by Lemma 1.1, there exist real numbers $A$ and $B$ $\left(A^{2}+B^{2} \neq 0\right)$, such that

$$
A \frac{x^{(p-1)(1-(\lambda / r))}}{y^{1-(\lambda / s)}} f^{p}(x)=B \frac{y^{(q-1)(1-(\lambda / s))}}{x^{1-(\lambda / r)}} g^{q}(y), \quad \text { a.e. in }(0, \infty) \times(0, \infty) .
$$

It follows that there exists a constant $D$, such that

$$
A x^{p(1-(\lambda / r))} f^{p}(x)=B y^{q(1-(\lambda / s))} g^{q}(y)=D, \quad \text { a.e. in }(0, \infty) \times(0, \infty) .
$$

Assume, without lose of generality, that $A \neq 0$, then we have

$$
x^{p(1-(\lambda / r))-1} f^{p}(x)=\frac{D}{A x}, \quad \text { a.e. in }(0, \infty),
$$

which contradicts with the facts that $f \in L_{\omega}^{p}\left(\mathbb{R}_{+}\right)$and $\|f\|_{p, \omega}>0$. Hence, (2.3) takes the form of strict inequality. So does (2.4). Then, we obtain (2.1).

Supposing there exists a number $0<C \leq C_{\lambda}(s)$, such that $(2.1)$ is still valid when $C_{\lambda}(s)$ is replaced by $C$, then for $n \in \mathbb{N}$, we have

$$
\begin{aligned}
\left(T f_{n}, g_{n}\right) & <C\left\{\int_{0}^{\infty} x^{p(1-(\lambda / r))-1} f_{n}^{p}(x) d x\right\}^{1 / p}\left\{\int_{0}^{\infty} y^{q(1-(\lambda / s))-1} g_{n}^{q}(y) d y\right\}^{1 / q} \\
& =C \int_{1}^{\infty} x^{-1-(1 / n)} d x=n C
\end{aligned}
$$


where

$$
\begin{aligned}
& f_{n}(x)= \begin{cases}x^{-(1-(\lambda / r))-(1 / n p)}, & x \in(1, \infty), \\
0, & x \in(0,1],\end{cases} \\
& g_{n}(y)= \begin{cases}y^{-(1-(\lambda / s))-(1 / n p)}, & y \in(1, \infty), \\
0, & y \in(0,1] .\end{cases}
\end{aligned}
$$

But by (1.15) and (1.16), we have

$$
\begin{aligned}
\left(T f_{n}, g_{n}\right) & =\iint_{0}^{\infty} K(x, y) f_{n}(x) g_{n}(y) d x d y \\
& =I_{n}=n\left[\int_{0}^{1} K(1, u) u^{(\lambda / s)-1+(1 / n p)} d u+\int_{1}^{\infty} K(1, u) u^{(\lambda / s)-1-(1 / n q)} d u\right] .
\end{aligned}
$$

In view of (2.8), we have

$$
\int_{0}^{1} K(1, u) u^{(\lambda / s)-1+(1 / n p)} d u+\int_{1}^{\infty} K(1, u) u^{(\lambda / s)-1-(1 / n q)} d u \leq C .
$$

Then by Fatou lemma [14], we have

$$
\begin{aligned}
C_{\lambda}(s) & =\int_{0}^{\infty} K(1, u) u^{(\lambda / s)-1} d u \\
& =\int_{0}^{1} \lim _{n \rightarrow \infty} K(1, u) u^{(\lambda / s)-1+(1 / n p)} d u+\int_{1}^{\infty} \lim _{n \rightarrow \infty} K(1, u) u^{(\lambda / s)-1-(1 / n q)} d u \\
& \leq \lim _{n \rightarrow \infty}\left[\int_{0}^{1} K(1, u) u^{(\lambda / s)-1+(1 / n p)} d u+\int_{1}^{\infty} K(1, u) u^{(\lambda / s)-1-(1 / n q)} d u\right] \leq C .
\end{aligned}
$$

Hence, the constant factor $C=C_{\lambda}(s)$ is the best possible.

(2) Setting $g(y)=y^{(p \lambda / s)-1}\left(\int_{0}^{\infty} K(x, y) f(x) d x\right)^{p-1}(y \in(0, \infty))$, then we have $g(y) \geq 0$. Using the notation (1.3), by Hölder's inequality (1.10) (as in (2.3)), we have

$$
\begin{aligned}
\|T f\|_{p, h}^{p} & =\|g\|_{q, \varpi}^{q}=\int_{0}^{\infty} y^{q(1-(\lambda / s))-1} g^{q}(y) d y \\
& =\int_{0}^{\infty} y^{(p \lambda / s)-1}\left(\int_{0}^{\infty} K(x, y) f(x) d x\right)^{p} d y=(T f, g) \leq C_{\lambda}(s)\|f\|_{p, \omega}\|g\|_{q, \varpi,}
\end{aligned}
$$

which is equivalent to

$$
\|T f\|_{p, h}^{p}=\|g\|_{q, \omega}^{q} \leq C_{\lambda}^{p}(s)\|f\|_{p, w}^{p} .
$$

In view of $f \in L_{\omega}^{p}\left(\mathbb{R}_{+}\right),\|f\|_{p, \omega}>0$, and condition (2), it follows that $T f \in L_{h}^{p}\left(\mathbb{R}_{+}\right), g \in L_{\varpi}^{q}\left(\mathbb{R}_{+}\right)$, and $\|g\|_{q, \varpi}>0$. Using (2.1), we can find that inequality (2.13) takes the form of strict inequality. So does (2.14). Hence, we obtain (2.2). 
On the other hand, if inequality (2.2) holds, then by using Hölder's inequality (1.10) again, we find

$$
\begin{aligned}
(T f, g) & =\iint_{0}^{\infty} K(x, y) f(x) g(y) d x d y \\
& =\int_{0}^{\infty}\left[y^{(\lambda / s)-(1 / p)} \int_{0}^{\infty} K(x, y) f(x) d x\right]\left[y^{(1 / p)-(\lambda / s)} g(y)\right] d y \\
& \leq\left\{\int_{0}^{\infty} y^{(p \lambda / s)-1}\left(\int_{0}^{\infty} K(x, y) f(x) d x\right)^{p} d y\right\}^{(1 / p)}\left\{\int_{0}^{\infty} y^{q(1-(\lambda / s))-1} g^{q}(y) d y\right\}^{1 / q} .
\end{aligned}
$$

By (2.2), we have (2.1). It follows that (2.2) is equivalent to (2.1). If the constant factor $C_{\lambda}(s)$ in (2.2) is not the best possible, then by (2.15), we can get a contradiction that the constant factor $C_{\lambda}(s)$ in (2.1) is not the best possible. The theorem is proved.

Theorem 2.2. Let $0<p<1(q<0),(1 / p)+(1 / q)=1, r>1,(1 / r)+(1 / s)=1, \lambda>0$. Assume that $f, g \geq 0, \omega(x), \varpi(y), h(y)$, and the integral operator $T$ are all defined as in Theorem 2.1. Setting $\phi(x)=x^{(q \lambda / r)-1}$ if

(1) $K(x, y)$ is a nonnegative measurable homogeneous kernel function of- $\lambda$-degree,

(2) the weight coefficient $C_{\lambda}(s)=\int_{0}^{\infty} K(1, u) u^{(\lambda / s)-1} d u$ is a positive number depending only on the parameters $\lambda, s$, then one has the following cases:

if $f \in L_{\omega}^{p}\left(\mathbb{R}_{+}\right), g \in L_{\varpi}^{q}\left(\mathbb{R}_{+}\right)$, and $\|f\|_{p, \omega},\|g\|_{q, \varpi}>0$, then

$$
(T f, g)=\iint_{0}^{\infty} K(x, y) f(x) g(y) d x d y>C_{\lambda}(s)\|f\|_{p, \omega}\|g\|_{q, \varpi},
$$

if $f \in L_{\omega}^{p}\left(\mathbb{R}_{+}\right)$and $\|f\|_{p, \omega}>0$, then

$$
\|T f\|_{p, h}=\left\{\int_{0}^{\infty} y^{(p \lambda / s)-1}\left(\int_{0}^{\infty} K(x, y) f(x) d x\right)^{p} d y\right\}^{1 / p}>C_{\lambda}(s)\|f\|_{p, \omega},
$$

if $g \in L_{\varpi}^{q}\left(\mathbb{R}_{+}\right)$and $\|g\|_{q, \varpi}>0$, then $T g \in L_{\phi}^{q}\left(\mathbb{R}_{+}\right)$, and

$$
\|T g\|_{q, \phi}^{q}=\int_{0}^{\infty} x^{(q \lambda / r)-1}\left(\int_{0}^{\infty} K(x, y) g(y) d y\right)^{q} d x<C_{\lambda}^{q}(s)\|g\|_{q, w}^{q},
$$

where the constant factors $C_{\lambda}(s)$ and $C_{\lambda}^{q}(s)$ in (2.16) (or in (2.17)) and in (2.18), respectively, are both the best possible. Inequalities (2.17) and (2.18) are both equivalent to inequality (2.16).

Proof. (1) Since $0<p<1$, we can use the reverse Hölder inequality (1.11). Using the combination as in (2.3) and using the notations (1.9) and (1.10), we have

$$
\begin{aligned}
(T f, g) & =\iint_{0}^{\infty}\left[K^{1 / p}(x, y) f(x) \frac{x^{(1 / q)(1-(\lambda / r))}}{y^{(1 / p)(1-(\lambda / s))}}\right]\left[K^{1 / q}(x, y) g(y) \frac{y^{(1 / p)(1-(\lambda / s))}}{x^{(1 / q)(1-(\lambda / r))}}\right] d x d y \\
& \geq\left\{\int_{0}^{\infty} \omega_{1}(\lambda, s, x) x^{p(1-(\lambda / r))-1} f^{p}(x) d x\right\}^{1 / p}\left\{\int_{0}^{\infty} \omega_{2}(\lambda, r, y) y^{q(1-(\lambda / s))-1} g^{q}(y) d y\right\}^{1 / q} .
\end{aligned}
$$


By (1.12), condition (2), and notation (1.3), we have

$$
(T f, g) \geq C_{\lambda}(s)\|f\|_{p, \omega}\|g\|_{q, w} .
$$

If (2.19) takes the form of equality. Then by using the conclusions of (2.5)-(2.7), we still can get a result which contradicts the conditions of $f \in L_{\omega}^{p}\left(\mathbb{R}_{+}\right)$and $\|f\|_{p, \omega}>0$ (or $g \in L_{\varpi}^{q}\left(\mathbb{R}_{+}\right)$and $\left.\|g\|_{q, \varpi}>0\right)$. It means that (2.19) takes the form of strict inequality. So does (2.20). The form (2.16) is valid.

If there exists a positive number $C \geq C_{\lambda}(s)$, such that (2.16) is still valid when $C_{\lambda}(s)$ is replaced by $C$, then for $n \in \mathbb{N}$, setting $f_{n}(x)$ and $g_{n}(y)$ as (2.9), we have

$$
\left(T f_{n}, g_{n}\right)>C\left\|f_{n}\right\|_{p, \omega}\left\|g_{n}\right\|_{q, \varpi}=C \int_{1}^{\infty} x^{-1-(1 / n)} d x=n C .
$$

But by (1.16), we have

$$
\begin{aligned}
\left(T f_{n}, g_{n}\right) & =\iint_{0}^{\infty} k(x, y) f_{n}(x) g_{n}(y) d x d y \\
& =I_{n}=n\left[\int_{0}^{1} K(1, u) u^{(\lambda / s)-1+(1 / n q)} d u+\int_{1}^{\infty} K(1, u) u^{(\lambda / s)-1-(1 / n q)} d u\right] .
\end{aligned}
$$

In view of (2.21), we have

$$
C<\int_{0}^{1} K(1, u) u^{(\lambda / s)-1+(1 / n p)} d u+\int_{1}^{\infty} K(1, u) u^{(\lambda / s)-1-(1 / n q)} d u .
$$

Also by the nonnegative measurability of $K(x, y)$, we can use Fatou lemma and obtain

$$
\begin{aligned}
C & \leq \lim _{n \rightarrow \infty}\left[\int_{0}^{1} K(1, u) u^{(\lambda / s)-1+(1 / n p)} d u+\int_{1}^{\infty} K(1, u) u^{(\lambda / s)-1-(1 / n q)} d u\right] \\
& =\int_{0}^{1} \lim _{n \rightarrow \infty} K(1, u) u^{(\lambda / s)-1+(1 / n p)} d u+\int_{1}^{\infty} \lim _{n \rightarrow \infty} K(1, u) u^{(\lambda / s)-1-(1 / n q)} d u=C_{\lambda}(s) .
\end{aligned}
$$

Hence, the constant factor $C=C_{\lambda}(s)$ is the best possible.

(2) Setting $g(y)=y^{(p \lambda / s)-1}\left(\int_{0}^{\infty} K(x, y) f(x) d x\right)^{p-1}(y \in(0, \infty))$, we have $g(y) \geq 0$. By the notation (1.3) and in view of (2.19), we have

$$
\begin{aligned}
\|T f\|_{p, h}^{p} & =\|g\|_{q, \varpi}^{q}=\int_{0}^{\infty} y^{q(1-(\lambda / s))-1} g^{q}(y) d y \\
& =\int_{0}^{\infty} y^{(p \lambda / s)-1}\left(\int_{0}^{\infty} K(x, y) f(x) d x\right)^{p} d y=(T f, g) \geq C_{\lambda}(s)\|f\|_{p, \omega}\|g\|_{q, \varpi}, \\
\|T f\|_{p, h}^{p} & =\|g\|_{q, \varpi}^{q} \geq C_{\lambda}^{p}(s)\|f\|_{p, \omega}^{p} .
\end{aligned}
$$

If $\|T f\|_{p, h}^{p}=\|g\|_{q, \omega}^{q}=\infty$, then by $f \in L_{\omega}^{p}\left(\mathbb{R}_{+}\right),(2.25)$ takes the form of strict inequality, so does (2.26). That is what (2.17) holds. If $T f \in L_{h}^{p}\left(\mathbb{R}_{+}\right)\left(g \in L_{\varpi}^{q}\left(\mathbb{R}_{+}\right)\right)$, it tells us that the conditions of 
(2.16) are satisfied. Then by using (2.16), it follows that both (2.25) and (2.26) keep the forms of strict inequality and (2.17) holds.

On the other hand, if (2.17) is valid, then by using the reverse Hölder inequality (1.11) again, we have

$$
\begin{aligned}
(T f, g) & =\int_{0}^{\infty} y^{(\lambda / s)-(1 / p)}\left[\int_{0}^{\infty} K(x, y) f(x) d x\right]\left[y^{(1 / p)-(\lambda / s)} g(y)\right] d y \\
& \geq\left\{\int_{0}^{\infty} y^{(p \lambda / s)-1}\left[\int_{0}^{\infty} K(x, y) f(x) d x\right]^{p} d y\right\}^{1 / p}\left\{\int_{0}^{\infty} y^{q(1-(\lambda / s))-1} g^{q}(y) d y\right\}^{1 / q} .
\end{aligned}
$$

By (2.17), we have (2.16). It means that (2.17) is equivalent to (2.16).

(3) Firstly, setting $f(x)=x^{(q \lambda / r)-1}\left(\int_{0}^{\infty} K(x, y) g(y) d y\right)^{q-1}(x \in(0, \infty))$, then we have $f(x) \geq 0$. Using the notation (1.3) and in view of (2.19), (2.20), we have

$$
\begin{aligned}
\|T g\|_{q, \phi}^{q} & =\|f\|_{p, \omega}^{p}=\int_{0}^{\infty} x^{p(1-(\lambda / r))-1} f^{p}(x) d x \\
& =\int_{0}^{\infty} x^{(q \lambda / r)-1}\left(\int_{0}^{\infty} K(x, y) g(y) d y\right)^{q} d y=(T g, f) \geq C_{\lambda}(s)\|f\|_{p, \omega}\|g\|_{q, \omega} .
\end{aligned}
$$

It follows that

$$
\|T g\|_{q, \phi}=\|f\|_{p, \omega}^{p / q}=\left\{\int_{0}^{\infty} x^{p(1-(\lambda / r))-1} f^{p}(x) d x\right\}^{1 / q} \geq C_{\lambda}(s)\|g\|_{q, \varpi},
$$

and by $q<0$ (because of $0<p<1$ ), we have

$$
0<\|T g\|_{q, \phi}^{q}=\|f\|_{p, \omega}^{p}=\int_{0}^{\infty} x^{(q \lambda / r)-1}\left(\int_{0}^{\infty} K(x, y) g(y) d y\right)^{q} d y \leq C_{\lambda}^{q}(s)\|g\|_{q, \omega}^{q}<\infty .
$$

It follows that $T g \in L_{\phi}^{q}\left(\mathbb{R}_{+}\right), f \in L_{\omega}^{p}\left(\mathbb{R}_{+}\right)$. Also by (2.16), we find that (2.28) and (2.30) are strict inequalities. Thus, inequality (2.18) holds.

Secondly, if (2.18) is valid, then by using the reverse Hölder inequality (1.11) again, in view of

$$
\begin{aligned}
(T f, g) & =\iint_{0}^{\infty} K(x, y) f(x) g(y) d x d y \\
& =\int_{0}^{\infty}\left[x^{(1 / q)-(\lambda / r)} f(x)\right]\left[x^{(\lambda / r)-(1 / q)} \int_{0}^{\infty} K(x, y) g(y) d y\right] d x \\
& \geq\left\{\int_{0}^{\infty} x^{p(1-(\lambda / r))-1} f^{p}(x) d x\right\}^{1 / p}\left\{\int_{0}^{\infty} x^{(q \lambda / r)-1}\left[\int_{0}^{\infty} K(x, y) g(y) d y\right]^{q} d x\right\}^{1 / q},
\end{aligned}
$$

by (2.18) and $q<0$, we can easily find that (2.16) holds, and (2.18) is equivalent to (2.16).

If the constant factor $C_{\lambda}(s)$ ( or $C_{\lambda}^{q}(s)$ ) in (2.17) (or in (2.18)) is not the best possible, then by (2.27) (or (2.31)), we can get a contradiction that the constant factor $C_{\lambda}(s)$ in (2.16) is not the best possible. The theorem is proved. 


\section{Some particular cases}

Corollary 3.1. Let $p>0,(1 / p)+(1 / q)=1, r>1,(1 / r)+(1 / s)=1, \lambda>0, \omega(x)=$ $x^{p(1-(\lambda / r))-1}, \varpi(y)=y^{q(1-\lambda / s)-1}$ and $f, g \geq 0$, then one has the following cases: if $p>1, f \in$ $L_{\omega}^{p}\left(\mathbb{R}_{+}\right), g \in L_{\varpi}^{q}\left(\mathbb{R}_{+}\right)$, and $\|f\|_{p, \omega},\|g\|_{q, \varpi}>0$, then

$$
\iint_{0}^{\infty} \frac{x^{\curlywedge} f(x) g(y)}{\left(x^{\curlywedge}+y^{\curlywedge}\right) \max \left\{x^{\curlywedge}, y^{\curlywedge}\right\}} d x d y<C_{\lambda}(s)\|f\|_{p, \omega}\|g\|_{q, \varpi},
$$

if $p>1, f \in L_{\omega}^{p}\left(\mathbb{R}_{+}\right)$, and $\|f\|_{p, \omega}>0$, then

$$
\int_{0}^{\infty} y^{(p \lambda / s)-1}\left(\int_{0}^{\infty} \frac{x^{\lambda} f(x)}{\left(x^{\lambda}+y^{\lambda}\right) \max \left\{x^{\lambda}, y^{\lambda}\right\}} d x\right)^{p} d y<C_{\lambda}^{p}(s)\|f\|_{p, \omega}^{p},
$$

if $0<p<1, f \in L_{\omega}^{p}\left(\mathbb{R}_{+}\right), g \in L_{\varpi}^{q}\left(\mathbb{R}_{+}\right)$, and $\|f\|_{p, \omega},\|g\|_{q, \varpi}>0$, then

$$
\iint_{0}^{\infty} \frac{x^{\lambda} f(x) g(y)}{\left(x^{\curlywedge}+y^{\lambda}\right) \max \left\{x^{\curlywedge}, y^{\lambda}\right\}} d x d y>C_{\lambda}(s)\|f\|_{p, \omega}\|g\|_{q, \varpi},
$$

if $0<p<1, f \in L_{\omega}^{p}\left(\mathbb{R}_{+}\right)$, and $\|f\|_{p, \omega}>0$, then

$$
\int_{0}^{\infty} y^{(p \jmath / s)-1}\left(\int_{0}^{\infty} \frac{x^{\lambda} f(x)}{\left(x^{\curlywedge}+y^{\lambda}\right) \max \left\{x^{\curlywedge}, y^{\lambda}\right\}} d x\right)^{p} d y>C_{\lambda}^{p}(s)\|f\|_{p, \omega}^{p},
$$

if $0<p<1, g \in L_{\varpi}^{q}\left(\mathbb{R}_{+}\right)$, and $\|g\|_{q, \varpi}>0$, then

$$
\int_{0}^{\infty} x^{(q \lambda / r)-1}\left(\int_{0}^{\infty} \frac{x^{\lambda} g(y)}{\left(x^{\lambda}+y^{\lambda}\right) \max \left\{x^{\curlywedge}, y^{\lambda}\right\}} d y\right)^{q} d x<C_{\lambda}^{q}(s)\|g\|_{q, \varpi}^{q},
$$

where the constant factors $C_{\lambda}(s)=(2 / \lambda) \sum_{k=0}^{\infty}\left((-1)^{k}(k+1)\right) /((k+(1 / r)+1)(k+(1 / s))), C_{\lambda}^{p}(s)$ and $C_{\lambda}^{q}(s)$ are all the best possible. Inequality (3.2) is equivalent to (3.1), and inequalities (3.4) and (3.5) are both equivalent to (3.3).

Proof. Let $K(x, y)=x^{\lambda} /\left(\left(x^{\lambda}+y^{\lambda}\right) \max \left\{x^{\lambda}, y^{\lambda}\right\}\right)$, then $K(x, y)$ is a nonnegative measurable homogeneous kernel function of $-\lambda$-degree in $(0, \infty) \times(0, \infty)$. In view of Theorems 2.1 and 2.2, we only need to show that

$$
C_{\lambda}(s)=\int_{0}^{\infty} k(1, u) u^{(\lambda / s)-1} d u
$$

is a positive number depending only on the parameters $\lambda, s$.

Setting $t=u^{\lambda}$, we have

$$
\begin{aligned}
C_{\lambda}(s) & =\int_{0}^{\infty} k(1, u) u^{(\lambda / s)-1} d u=\int_{0}^{\infty} \frac{u^{(\lambda / s)-1}}{\left(1+u^{\lambda}\right) \max \left\{1, u^{\lambda}\right\}} d u \\
& =\frac{1}{\lambda}\left[\int_{0}^{1} \frac{t^{(1 / s)-1}}{1+t} d t+\int_{1}^{\infty} \frac{t^{(1 / s)-1}}{(1+t) t} d t\right]=\frac{1}{\lambda}\left(H_{1}+H_{2}\right) .
\end{aligned}
$$


By $a=(1 / s)>0$, we find that $H_{1}=\int_{0}^{1}\left(t^{(1 / s)-1} /(1+t)\right) d t$ is a Eulerian integral [15] and we have

$$
H_{1}=\sum_{k=0}^{\infty} \frac{(-1)^{k}}{k+(1 / s)}
$$

Also letting $v=1 / t$ in $H_{2}$, we also have

$$
H_{2}=\int_{0}^{1} \frac{v^{(1 / r)}}{1+v} d v=\sum_{k=0}^{\infty} \frac{(-1)^{k}}{k+(1 / r)+1}
$$

In view of (3.8) and (3.9), we have $C_{\lambda}(s)=(2 / \lambda) \sum_{k=0}^{\infty}\left((-1)^{k}(k+1)\right) /((k+(1 / r)+1)(k+(1 / s)))$. It shows that $C_{\lambda}(s)$ is a positive number. By Theorems 2.1 and 2.2, Corollary 3.1 is proved.

Corollary 3.2. Suppose that $p>0,(1 / p)+(1 / q)=1, r>1,(1 / r)+(1 / s)=1, \lambda>0, \omega(x)=$ $x^{p(1-(\lambda / r))-1}, \varpi(y)=y^{q(1-(\lambda / s))-1}$, and $f, g \geq 0$. One has the following cases:if $p>1, f \in L_{\omega}^{p}\left(\mathbb{R}_{+}\right), g \in$ $L_{\varpi}^{q}\left(\mathbb{R}_{+}\right)$, and $\|f\|_{p, \omega},\|g\|_{q, \varpi}>0$, then

$$
\iint_{0}^{\infty} \frac{x^{\curlywedge} f(x) g(y)}{\left(x^{2 \lambda}+y^{2 \lambda}\right)+\left|x^{2 \lambda}-y^{2 \lambda}\right|} d x d y<C_{\lambda}(s)\|f\|_{p, \omega}\|g\|_{q, \varpi}
$$

if $p>1, f \in L_{\omega}^{p}\left(\mathbb{R}_{+}\right)$, and $\|f\|_{p, \omega}>0$, then

$$
\int_{0}^{\infty} y^{(p \lambda / s)-1}\left(\int_{0}^{\infty} \frac{x^{\lambda} f(x)}{\left(x^{2 \lambda}+y^{2 \lambda}\right)+\left|x^{2 \lambda}-y^{2 \lambda}\right|} d x\right)^{p} d y<C_{\lambda}^{p}(s)\|f\|_{p, \omega}^{p}
$$

if $0<p<1, f \in L_{\omega}^{p}\left(\mathbb{R}_{+}\right), g \in L_{\varpi}^{q}\left(\mathbb{R}_{+}\right)$, and $\|f\|_{p, \omega},\|g\|_{q, \varpi}>0$, then

$$
\iint_{0}^{\infty} \frac{x^{\lambda} f(x) g(y)}{\left(x^{2 \lambda}+y^{2 \lambda}\right)+\left|x^{2 \lambda}-y^{2 \lambda}\right|} d x d y>C_{\lambda}(s)\|f\|_{p, \omega}\|g\|_{q, \varpi}
$$

if $0<p<1, f \in L_{\omega}^{p}\left(\mathbb{R}_{+}\right)$, and $\|f\|_{p, \omega}>0$, then

$$
\int_{0}^{\infty} y^{(p \lambda / s)-1}\left(\int_{0}^{\infty} \frac{x^{\lambda} f(x)}{\left(x^{2 \lambda}+y^{2 \lambda}\right)+\left|x^{2 \lambda}-y^{2 \lambda}\right|} d x\right)^{p} d y>C_{\lambda}^{p}(s)\|f\|_{p, \omega}^{p}
$$

if $0<p<1, g \in L_{\varpi}^{q}\left(\mathbb{R}_{+}\right)$, and $\|g\|_{q, \varpi}>0$, then

$$
\int_{0}^{\infty} x^{(q \lambda / r)-1}\left(\int_{0}^{\infty} \frac{x^{\lambda} g(y)}{\left(x^{2 \lambda}+y^{2 \lambda}\right)+\left|x^{2 \lambda}-y^{2 \lambda}\right|} d y\right)^{q} d x<C_{\lambda}^{q}(s)\|g\|_{q, \varpi}^{q},
$$

where the constant factor $C_{\lambda}(s)=(s r) /(\lambda(r+1)), C_{\lambda}^{p}(s)$ and $C_{\lambda}^{q}(s)$ are all the best possible. Inequality (3.11) is equivalent to (3.10) and inequalities (3.13) and (3.14) are both equivalent to (3.12). 
Proof. Setting $K(x, y)=x^{\lambda} /\left(\left(x^{2 \lambda}+y^{2 \lambda}\right)+\left|x^{2 \lambda}-y^{2 \lambda}\right|\right)$, then $K(x, y)$ is a nonnegative measurable homogeneous kernel function of $-\lambda$-degree in $(0, \infty) \times(0, \infty)$. As in Corollary 3.1, we only need to show that $C_{\lambda}(s)$ is a positive number depending only on the parameters $\lambda, \mathrm{s}$.

Setting $t=u^{2 \lambda}$, for $p>0, p \neq 1$, we have

$$
\begin{aligned}
C_{\lambda}(s) & =\int_{0}^{\infty} K(1, u) u^{(\lambda / s)-1} d u=\int_{0}^{\infty} \frac{u^{(\lambda / s)-1}}{\left(1+u^{2 \lambda}\right)+\left|1-u^{2 \lambda}\right|} d u \\
& =\frac{1}{2 \lambda} \int_{0}^{\infty} \frac{t^{(1 / 2 s)-1}}{1+t+|1-t|} d t=\frac{1}{4 \lambda}\left[\int_{0}^{1} t^{(1 / 2 s)-1} d t+\int_{1}^{\infty} t^{(1 / 2 s)-2} d t\right]=\frac{s r}{\lambda(r+1)} .
\end{aligned}
$$

It shows that $C_{\lambda}(s)=s r /(\lambda(r+1))$ is a positive number. Corollary 3.2 is proved.

Remark 3.3. (1) The homogeneous kernel functions $K(x, y)$ appearing in Corollaries 3.1 and 3.2 are just two especial examples. We can give more other examples.

(2) We can extend Theorems 2.1 and 2.2 to higher-dimensional space $\mathbb{R}_{+}^{n} \times \mathbb{R}_{+}^{n}$ easily.

(3) Letting $\lambda=3, r=s=2$ in the forms (2.1), (2.2), (2.16), and (2.17), and setting $K(x, y)=1 /\left(\left(x+a^{2} y\right)\left(x+b^{2} y\right)\left(x+c^{2} y\right)\right)$, then we can get Xie's results [12] at once. So Xie's work is a special case of our Theorems 2.1 and 2.2.

\section{References}

[1] G. H. Hardy, J. E. Littlewood, and G. Pólya, Inequalities, Cambridge University Press, Cambridge, UK, 2nd edition, 1952.

[2] B. Yang, "On the norm of an integral operator and applications," Journal of Mathematical Analysis and Applications, vol. 321, no. 1, pp. 182-192, 2006.

[3] I. Brnetić and J. Pečarić, "Generalization of inequalities of Hardy-Hilbert type," Mathematical Inequalities \& Applications, vol. 7, no. 2, pp. 217-225, 2004.

[4] W. Zhong and B. Yang, "A best extension of Hilbert inequality involving seveial parameters," Journal of Jinan University (Natural Sciences and Medicine), vol. 28, no. 1, pp. 20-23, 2007.

[5] B. Yang and L. Debnath, "On the extended Hardy-Hilbert's inequality," Journal of Mathematical Analysis and Applications, vol. 272, no. 1, pp. 187-199, 2002.

[6] B. Yang and M. Gao, "An optimal constant in the Hardy-Hilbert inequality," Advances in Mathematics, vol. 26, no. 2, pp. 159-164, 1997 (Chinese).

[7] C. Zhao and L. Debnath, "Some new inverse type Hilbert integral inequalities," Journal of Mathematical Analysis and Applications, vol. 262, no. 1, pp. 411-418, 2001.

[8] W. Zhong, "A reverse Hilbert's type integral inequality," International Journal of Pure and Applied Mathematics, vol. 36, no. 3, pp. 353-360, 2007.

[9] W. Zhong and B. Yang, "On the extended form on the reverse Hardy-Hilbert's integral inequalities," Journal of Southwest China Normal University (Natural Science), vol. 29, no. 4, pp. 44-48, 2007.

[10] M. Krnić and J. Pečarić, "General Hilbert's and Hardy's inequalities," Mathematical Inequalities \& Applications, vol. 8, no. 1, pp. 29-51, 2005.

[11] W. Zhong and B. Yang, "On a multiple Hilbert-type integral inequality with the symmetric kernel," Journal of Inequalities and Applications, vol. 2007, Article ID 27962, 17 pages, 2007.

[12] Z. Xie and Z. Zheng, "A Hilbert-type integral inequality whose kernel is a homogeneous form of degree -3," Journal of Mathematical Analysis and Applications, vol. 339, no. 1, pp. 324-331, 2008.

[13] J. Kuang, Applied Inequalities, Shangdong Science and Technology Press, Jinan, China, 2004.

[14] J. Kuang, Real and Functional Analysis, Higher Education Press, Beijing, China, 2002.

[15] G. M. Fichtingoloz, A Course in Differential and Integral Calculus, Renmin Education Press, Beijing, China, 1978. 